\title{
The Effect Of Alpha-Element Enhancement On Surface Brightness Fluctuation Magnitudes And Broad-Band Colors
}

\author{
Hyun-chul Lee ${ }^{1}$, Guy Worthey ${ }^{1}$, and John P. Blakeslee ${ }^{1}$ \\ ${ }^{1}$ Washington State University \\ email: hclee@wsu.edu
}

\begin{abstract}
We present the effects due to alpha-element enhancement on surface brightness fluctuation magnitudes and broad-band colors in order to investigate the calibration zero-point. We study these effects at ages covering 0.1 to $13 \mathrm{Gyr}$ and metallicities of $\mathrm{Z}=0.0003$ to 0.04 using the Teramo group's isochrones, BaSTI. Our preliminary results indicate that alpha-enhanced models are needed to match massive red galaxies while solar-scaled models are fine for dwarf blue galaxies to reproduce their independently estimated ages and metallicities.
\end{abstract}

Keywords. Stars: abundances, galaxies: stellar content

\section{Introduction}

The surface brightness fluctuation (SBF) magnitudes and colors are widely used as one of the most powerful distance indicators as well as useful tools for probing stellar populations. To our continued consternation, however, only solar-scaled SBF models have been calculated in the past.

\section{Our Preliminary Results}

Here we report, for the first time, alpha-element enhanced SBF models and compare them with observations. For those purposes, we have employed the Teramo Isochrones (Pietrinferni et al. 2004, 2006; Cordier et al. 2007; http://www.te.astro.it/ BASTI ) in this study. In general, alpha-element enhanced SBF magnitudes are brighter and their broad-band colors are redder where the alpha-element enhancement is defined at a fixed total metallicity (cf., http:// www.astro.rug.nl/ sctrager/FTSPM/ Lee_alpha.pdf ). Our results also illustrate the dramatic impact of convective core overshooting on the predicted broad-band colors for models with ages near 1 Gyr (see also http://astro.wsu.edu/hclee/ hclee_208_AAS_10_07.pdf ).

Figure 1 shows our preliminary results (aes: alpha-enhanced standard, sss: solar-scaled standard, where 'standard' means no convective core overshooting) in the V - I vs. SBF I-band Magnitude. Two observational fiducial lines (thick straight lines) are overlaid with our theoretical models. The blue line is SBF I-band Mag $=-2.13+2.44 \times[(\mathrm{V}-\mathrm{I})-$ 1.00] from Mieske, Hilker, \& Infante (2006) for dwarf blue galaxies, while the red line is SBF I-band Mag $=-1.68+4.5 \times[(\mathrm{V}-\mathrm{I})-1.15]$ from Mei et al. (2005, originally calibrated at Blakeslee et al. 2002) for massive red galaxies. From the comparisons, it seems that our preliminary results indicate that alpha-enhanced models are needed to match massive red galaxies while solar-scaled models are fine for dwarf blue galaxies to reproduce their independently estimated ages and metallicities. The full investigation will be presented at Lee, Worthey, \& Blakeslee (2007). 


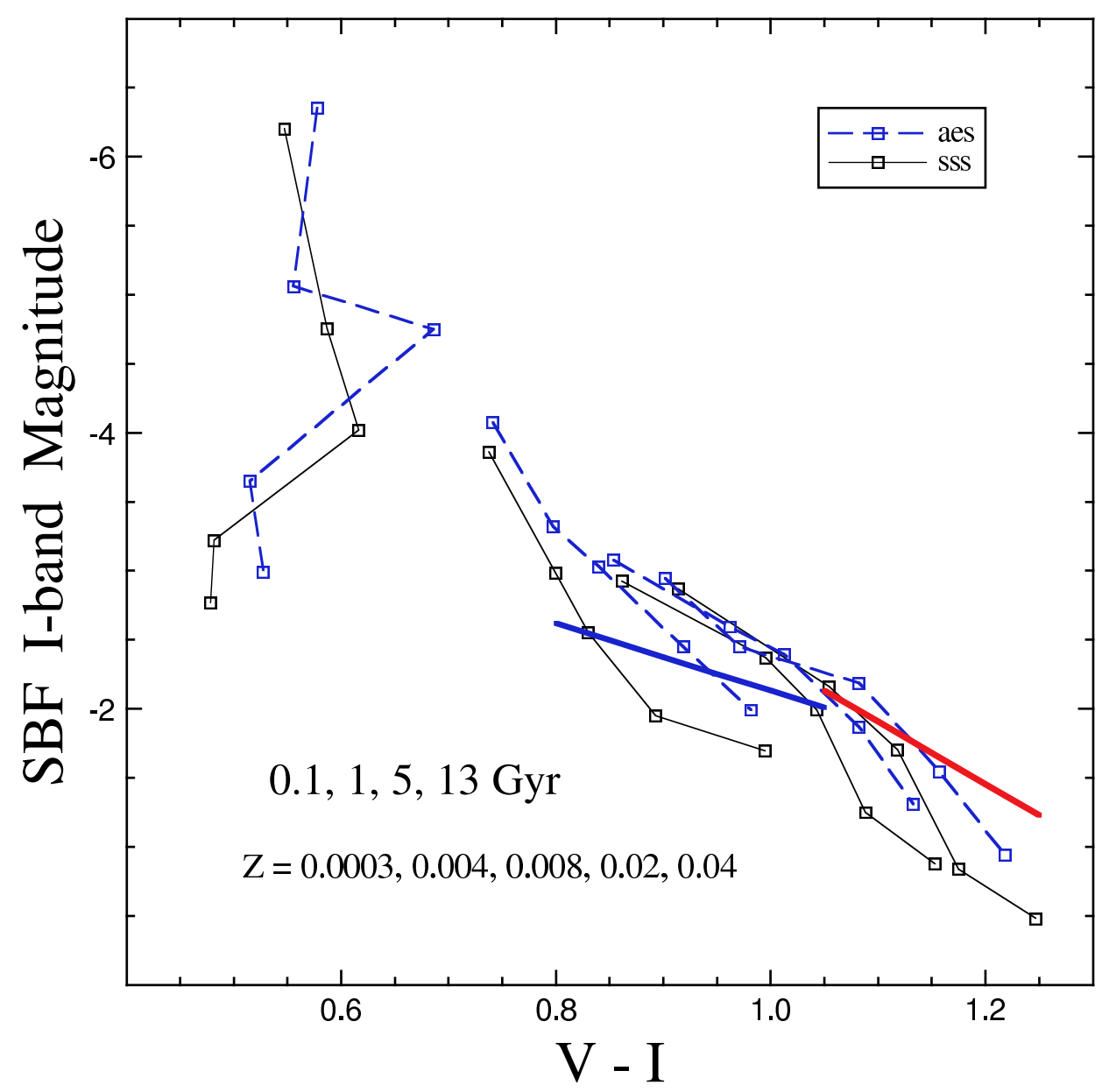

Figure 1. V - I vs. SBF I-band Magnitude at 0.1, 1, 5, and 13 Gyr (left to right). Solid lines with squares are solar-scaled models while dashed lines with squares are alpha-enhanced models. At given ages, SBF I-band Magnitudes become fainter and V - I colors become redder, in general, with increasing metallicity. Two observational fiducial lines are compared with our theoretical models (see text).

\section{Acknowledgements}

Support for this work was provided by the NSF through grant AST-0307487, the New Standard Stellar Population Models (NSSPM) project. H.-c. Lee also thanks the organizers for their wonderful hospitality and financial support.

\section{References}

Blakeslee, J.P., Lucey, J.R., Tonry, J.L. et al. 2002, MNRAS , 330, 443

Cordier, D., Pietrinferni, A., Cassisi, S., \& Salaris, M. 2007, AJ , 133, 468

Mei, s., Blakeslee, J.P., Tonry, J.L. et al. 2005, ApJ , 625, 121

Mieske, S., Hilker, M., \& Infante, L. 2006, A\&A A, 458, 1013

Pietrinferni, A., Cassisi, S., Salaris, M., \& Castelli, F. 2004, ApJ , 612, 168

Pietrinferni, A., Cassisi, S., Salaris, M., \& Castelli, F. 2006, ApJ , 642, 797 